\title{
Der Lautschwund im Ungarischen
}

Die Laute der verschiedenen Sprachen können im Lauf ihrer Geschichte qualitäts- und quantitätsmässige Veränderungen erfahren. Diese phonetischen Veränderungen verlaufen nach verschiedenen Gesetzmässigkeiten; sie stehen jedoch immer unter der regulierenden Herrschaft des gesamten inneren Systems der Sprache. Die Aufdeckung der Ursachen der einzelnen Lautveränderungen ist kein leichter Vorgang. Als Hindernis treten zwei Faktoren auf: einerseits kennen wir das zur Zeit der Veränderung wirkende innere System nicht genug, andererseits kennen wir nicht die vollständige Zahl derjenigen Sprachelemente, in denen sich die Veränderungen vollzogen. Diese Erfahrung machen wir auch bei der Untersuchung über das Verschwinden und Ausfallen von Isiuten. Es gibt nämlich Lautausfälle, die im Ungarischen fast konsequent auftreten, wie zum Beispiel der Schwund der Stammauslautvokale in der Ur- und Altzeit der Ungarn; es gibt dagegen auch Veränderungen, die nur auf einem engem Gebiet und nur vereinzelt zur Geltung gelangten.

Im folgenden konzentrieren wir uns auf quantitätsmässige phonetische Veränderungen, die nicht in allen solchen Wörtern erfolgten, in denen phonetische Bedingungen für die Veränderungen wenigstens scheinbar vorhanden waren. Wir betrachten in erster Linie das Schwinden von Vokalen und Konsonanten: inbegriffen sind Erscheinungen, die den İbergang zu den qualitätsmässigen Veränderungen bilden, wie z.B. die Vokalisierungr der Konsonanten, da deren Schicksal dennoch ein vollständiger Schwund war. Es ist richtig, dass nach der Vokalisierung von Konsonanten die Länge des Vokalelements 
das "quantitätsmässige» Element bewahrt; in einer Hinsicht hat es sich jedoch wesentlich verändert, da es seinen Konsonantencharakter verloren hat, was unter anderem auch darin zum Ausdruck kommt, dass es früher als Silbenschluss fungierte. Auf die Untersuchung der übrigen Erscheinungen quantitätsmässiger Änderungen (Verkürzung der Laute, Vereinfachung der Diphthonge) werden wir bei dieser Gelegenheit nicht eingehen.

Parallel zum U̇berblick über die Fälle von Lautausfall versuchen wir die den Lautausfall hervorrufenden Gründe aufzudecken; in diesem Forschungsbereich gibt es noch viel Unklares. Beim Aufdecken der Gründe für die Elision werden wir auch den Erscheinungen der Lauteinschiebung unsere Aufmerksamkeit widmen, ohne jedoch dafür veranschaulichende Belege zu bringen (vgl. jedoch MNy LVIII (1972) 75; MSFOu 150 (1973) 260-8). Lautausfall und Lauteinschiebung gehören nämlich oft sehr eng zusammen und sind häufig auf einund dieselbe Quelle zurückzuführen.

In absoluter Wortanfangs st ellung beobachten wir den Schwund der Laute $a-, z-; v-(\beta-), h-, j-$ und der als erstes Element der Konsonantenhäufungen auftretenden Laute $s$-, $s z-, v$ -

1. Das Verschwinden von $a$ - können wir in Wörtern sehen wie um 1405: patica, 1508: patikabeli (vgl. lat. apothica); 1558: lombekban, heute lombik 'Destillierblase, -kolben; Retorte' (vgl. lat. alembicum); 1597: lorant 'Nachmittagsmahlzeit, Vesperbrot, Jause' (vgl. slowak. olovrant, olevrant); 1789: bé-céket (TESz.), dial. bécéz, béce (vgl. a-bé-cé 'das ABC'); 1863: Lak (vgl. alak 'Form'); 1910-20: rabok (vgl. arab 'arabisch') usw.

Diese Erscheinung ist vereinzelt auch bis zum heutigen Tage in den Mundarten anzutreffen: jándék, jándok (vgl. ajándék 'Geschenk'), petitus (vgl. apetitus 'Appetit'), züte (vgl. azóta 'seitdem') usw. 
Ein ähnliches Schicksal hatte ganz gewiss auch das ungarische Wort napa 'Schwiegermutter' (vgl. wog. N. änip, finn. anoppi).

Der Grund für das Ausbleiben des Vokales $a$ - am Wortanfang ist durch Wortgrenzverwechs lu $\mathrm{ng}$, durch falsche Artikulation erklärbar. Die sprechenden Personen dürten den am Wortanfang befindlichen Laut, das $a-$, manchmal als bestimmten Artikel empfunden haben (z.B. apatika 'Apotheke' > a patika 'die Apotheke') und als solchen vom Wortanfang abgetrennt haben. Der anlautende Vokal des ungarischen Wortes napa ist nicht mit dem bestimmten Artikel, sondern mit dem vollständig gleichgestaltigen Demonstrativpronomen $a$ identifiziert worden (z.B. *anapa 'Schwiegermutter' > a napa 'die Schwiegermutter').

2. Eine ähnliche Erscheinung stellen wir auch bei den folgenden Wörtern fest, mit dem Unterschied, dass hier das $z$ am Wortanfang geschwunden ist: $a z a b>a z a b$ 'Hafer', a zabla >az abla 'Gebiss', a zászló >az ászló 'Fahne', zátony $>$ átony '(Sand)bank', zavadzál > acadzál 'schwätzen, quatschen', zacskó >acskó 'Tüte', (slaw. zháha >) záha >zaha $>$ aha 'Sodbrennen', zálog > álog 'Pfand' usw.

3. In bezug auf das Verschwinden der am Wortanfang befindlichen Laute $v$ - $(\beta$-) haben wir auch sehr alte Angaben. Bei einem Teil unserer Wörter, die finnisch-ugrischen Ursprungs sind und die Laute $v$ - $(\beta$-) im Wortanfang enthalten, ist der Laut $r$ - in der Regel geschwunden: ólom 'Blei' (vgl. wog. T wōlem), ón 'Zinn' (wog. T ålèn, tscher. KH $\beta u \cdot \ln \hat{a}$ usw.), orr 'Nase' (vgl. wog. T $\beta \overline{a r}$, ostj. V uor, finn. vuori usw.), öl 'töten' (vgl. wog. $\bar{a} l-$, ostj. wel-), öt 'fünf' (vgl. wog. $\bar{a} t$, ostj. wet, finn. viite-), heute visel > dial. üsel 'tragen' usw. (TESz., Hajdú, BUNy 107).

Das Verschwinden des Lautes $r$ - $(\beta$-) in den zitierten Angaben können wir mit E. Itkonen dadurch begründen, dass "vokaalien artikulaation ennakoitumisen johdosta ovat puolivokaalit $v$ ja $j$ voineet kadota niille artikulatorisesti läheisten vokaalien edeltä, esim. olla $<*$ vol- (vrt. unk. volt 'oli, on ollut')..." (Kieli ja sen tutkimus S. 165). Offenbar erfolgten ähnliche Veränderungen auch im Ungarischen. Das la- 
biale $v$ - $(\beta-)$ ist mit dem nachfolgenden, ebenfalls labialen Vokal verschmolzen, aber wenn dieser ursprünglich nicht labial war (z.B. finn. viite-), dann hat er im Hinblick auf die Labialisierung den Vokal assimiliert.

Das Verschwinden des $v$ - beschränkte sich jedoch nicht nur auf die phonetische Stellung vor dem labialen Vokal $(o, \ddot{o}<u, \ddot{u})$; auch vor den Lauten $i$ - und $e$ - konnte es verschwinden, ohne in irgendeiner Weise den hinter ihm stehenden Vokal zu assimilieren: z.B. um 1200: wimagguc (o: vimädd'uk 'beten wir!', HB.); 1201: vygor (o: vigor, Ortsname) > 1302: Igar puszta, 1348: Igor; um 1200: Velek (der Name der heutigen Gemeinde Elek); 1309: Wygman (Ortsname) $>I g-$ mánd; (slaw. vojsk > ung. *vojoszk $\sim$ vioszk >) éasz, iusz; (slaw. visák >) *viszák >iszák usw.

Das Verschwinden des $v$ - in den obigen Angaben können wir auf verschiedene Weise erklären. Nach sorgfältigem Erwägen bleiben jedoch vielleicht nur zwei Annahmen, zwischen denen wir wählen können. Es scheint auf der Hand zu liegen, das Schwinden des $v$ - vor den labialen Vokalen durch Analogiewirkung zu erklären und wir sagen, dass sich das Schwinden des $r$ - vor den labialen Vokalen auch auf diejenigen Wörter ausgedehnt hat, in denen die phonetischen Bedingungen geringfügig abweichend waren. Wir können jedoch nicht ausschliessen, dass das $v$ - durch einen Ausfall zwischen Vokalen an der Grenze zweier Wörter geschwunden ist.

4. Den Ausfall des Lautes $h$ - im Wortanfang können wir schon in einer kleinen Gruppe unserer Urwörter finnischugrischen Ursprungs beobachten. In diesen Wörtern geht das $h$ - manchmal auf das finnisch-ugrische ${ }^{*} s$ - zurück (z.B. ös $\sim$ wog. D tüks, tscher. šiža, finn. syksy; olvad 'schmelzen; tauen' $\sim$ wog. tol-, ostj. D tăt-, finn. sula usw.), manchmal aber auch auf das finmisch-ugrische *s- (z.B. egér 'Maus' wog. tänkar, ostj. D tenkar, finn. hïri; irt 'ausrotten; ausroden' ostj. V lŏrta, syrj. V širnì).

Das Schwinden des $h$ - im Wortanfang beschränkt sich jedoch nicht nur auf einen Teil unserer Wörter finnischugrischen Ursprungs, sondern ist auch bei unseren Wörtern 
deutschen, slawischen, (?) lateinischen usw. Ursprungs beobachtbar. Z.B. imper, impér 'Himbeere' (vgl. dt. K. himper), okusz-pókusz (vgl. internationales Wanderwort, mit deutscher Vermittlung Hokuspokus, ung. hókuszpókusz); 1493: offniczas, 1605: Ofincza 'Art Geschütz im Mittelalter' (vgl. tschech. houfnice); 1522: ymnus (vgl. lat. hymnus), istórija (vgl. lat. historia) usw. Der Ausfall des $h$-ist ferner auch bei solchen ungarischen Wörtern finnisch-ugrischen und autochthonen Ursprungs beobachtbar, bei denen wir nicht das finnisch-ugrische ${ }^{*} s$ - oder $*^{*} s$-, sondern den Laut $* k$ - im Wortanfang finden: z.B. hiába > jába 'unnötig, vergeblich' (vgl. ostj. Vj kŏm, tscher. KH koma.n, finn. komi, komo), hogy > dial. ogy, hogyan > dial. utyan (vgl. wog. T ķan 'ki?; wer?', ostj. V kòii 'ki?; wer?', finn. kuka usw.), hozzád > ozzád (vgl. 1522: the ozyad 'zu dir', zu seinem Ursprung vgl. ostj. V Trj

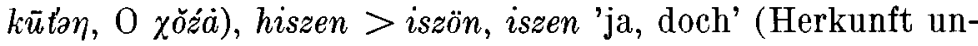
bekannt), hederint > ederint, (vgl. rá se ederéntöttem 'er achtete nicht einmal darauf'; eigenständiges Wort).

Die Ursache für den Schwund des $h$ - ist durch die bisherigen Forschungen überhaupt nicht geklärt worden. Hajdú stellt fest, dass das finnisch-ugrische ${ }^{*} s$ - und ${ }^{*}{ }^{-}$- über das ugrische $\vartheta$ verschwanden (BUNy 104-5). Bárczi weist darauf hin, dass "in der Regel die folgenden finnisch-ugrischen Konsonanten im Ungarischen ver s t u m m e n : Im Wortanfang fgr. ${ }^{*} s->$ ung. $\varnothing$; z.B.: ung. epe, vgl. wog. $t \bar{a} p$, finn. sappe-, wotj. sep usw.; ung. öl (Substantiv), vgl. ostj. lol, wog. täl, finn. syle-, syrj. sil usw. - Im Wortanfang fgr.

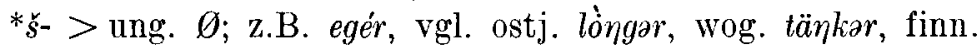
hiire-, syrj., wotj. šir usw.; ung. irt, vgl. ostj. lort-, syrj., wotj. šir-» (MNyT. 105). Er bemerkt ferner, dass wir nicht wissen, wann und auf welche Weise (durch $h$ ?) die vollständige Verstummung im Ungarischen eingetreten ist; ganz gewiss hat dieser Vorgang schon im Urungarischen seinen Abschluss gefunden (ebd.).

Die Aufklärung der Ursachen für das Versch wi nde $\mathrm{n}$ und das Verst um m n hat wegen des Mangels an sprachgeschichtlichen Angaben zu Hypothesen geführt: wir müssen annehmen, dass das $h$-im Wortanfang entweder 
auf physiologisch-phonetische Weise mit dem nachfolgenden Vokal verschmolzen ist, oder aber dass es an der Wortgrenze zwischen zwei Vokale gelangte und so ausgefallen ist.

Nach der urungarischen Zeit dürfte auch schon die Analogie beim Verschwinden des Lautes $h$ - im Wortanfang geholfen haben. Die geschichtlichen Daten bestätigen, dass es eine Zeit gab, da in der Sprache von Individuen in einzelnen Sprachbereichen ein und dasselbe Wort manchmal mit $h$ - und manchmal ohne $h$ - auftrat (z.B. 1240, 1319/1342: Herdeu э: herdeü $\sim 13 .-14$. Jahrhundert: Erdeu- o: erdeü 'Wald'; 1383: Heet-, nach 1416/um 1450: hèt $\sim$ um 1490: ethe 'sieben'; 1249: Eer o: ēr 1291: -heer o: hēr 'Wässerchen' usw.). Schwankungen solcher Art dürften sich später auch auf Formen ausgedehnt haben, in denen der Ausfall des $h$ - nicht zu erwarten war (vgl. oben jába, ogy, iszön).

5. Im absoluten Wortanlaut begegnen wir ferner auch dem Schwund des $j$ - in der urungarischen und auch in neuerer Zeit. Es ist allgemein bekannt, dass sich in einigen unserer Wörter finnisch-ugrischen Ursprungs das $j-(j-)$ vor dem $-i$ - abgeschliffen hat (vgl. finn. jänne $\sim$ ung. ideg; finn. jousi $\sim$ ung. $i j$, iv; finn. juoda $\sim$ ung. iszik usw., Hajdú, BUNy 107). Dieselbe Erscheinung ist auch bei unseren Wörtern jég 'Eis' nach 1416 /um 1490: egh; jérce 'junge Henne' érce $\sim$ érci; jegenye 'Pappel' > dial. egönye egenye; (türk.? *jyr-) > ung. ir; (slaw. jaremba >) ung. iromba 'bunt'; (slaw. *jatrocel >) ung. atracén atlacér 'Anchusa' usw. anzutreffen.

Das Schwinden des $j$ - im Wortanfang hat im Ungarischen zwei Gründe. Einerseits ist es dadurch erklärbar, dass das $j$ auf Grund seines Charakters den nachfolgenden tiefen Vokal palatalisierte und nach der Verschmelzung verschwand. Diese Veränderung ist dann analog auch auf andere Wörter übergegangen (s. ideg). Andererseits hat jedoch das $j$ - $(i-)$ im Wortanfang mit dem nachfolgenden palatalen Laut (z.B. $e, \ddot{e})$ einen Diphthong gebildet $(i e \sim i \vec{e} e, i e ́)$, wonach eine regelmässige Monophthongisierung erfolgte (z.B. érce).

6. Der Ausfall des ersten Elementes von Konsonantenhäufungen im Wortanlaut scheint im Ungarischen nicht häı- 
fig zu sein. Am häufigsten tritt er vielleicht in den Lautverbindungen $s t-, s k-, s z t^{-}, s z p-$ und $v l^{-}$auf: z.B. (lat. scolastica $>$) 1307: Korustica, 1320: kolustica (Frauenname); (dt. oder slowak. štiglinec >) 1320: Tengelych, dial. tiglic, tigline; (slaw. stoklas >) 1402: Toklasz; spenót > dial. penót, szputnyik > dial. putnyik, (slaw. Vladimir >) Ladomer, Lodomer; (slaw. Vladislav) László usw.

Das Ziel der sprachlichen Veränderungen ist das Beseitigen von Konsonantenhäufungen, die dem Lautsystem der ungarischen Sprache fremd sind; hier liegt ein Streben nach Energieeinsparung zugrunde.

\section{III}

1. Hinsichtlich der im Wortinneren erfolgenden Veränderungen wenden wir uns zunächst den in betonter Stellung eintretenden Ausfällen zu. Das zweite Element von Konsonantenhäufungen in Anfangsstellung kann ausfallen: das $l$ der Lautverbindung $f l-, k l-, s l$, das $r$ in $p r-, b r-, k r-, f r-$, das $v$ in $h v$-, $k v$ - und schliesslich das $t$ in der Lautverbindung szt-.

Bei eimer dreifachen Konsonantenhäufung fällt der mittlere Konsonant aus (slaw. stranzriko > zarándok).

Über Ursache und Zweck der Lautelision vgl. Abschnitt II/6 (vgl. auch Kálmán, MNyj II (1953) 59).

2. Der Ausfall von kurzen Vokalen $(e, a)$ in betonten Silben wird durch eine Gesetzmässigkeit hervorgerufen, die im Gegensatz zu den obigen gerade in entgegengesetzter Richtung wirkt. Die Veränderungen kalamáris > klamáris 'Tintenfass', parádé > prádé 'Parade', taráta > tráta 'überspannt, unordentlich', salétrom > slétrom 'Salpeter', teremtés > tremtés 'Schöpfung; Geschöpf', Terézia > Trézsi, Treszka usw. müssen wir als Gegensatz zur Auflösung von Konsonantenhäufungen, als sog. reziproke Lautentwicklung auffassen. Es ist nämlich allgemein bekannt, dass beim Eintreten einer sprachlichen Veränderung in einer gewissen Richtung auch die Umkehrung dieses Prozesses erfolgen kann. In den von 
uns erwähnten Beispielen stellen wir gerade das Wirken dieser Gesetzmässigkeit fest.

3. Ein sehr häufiger Fall von Lautschwund im Wortinnern und am Wortende ist der in einer geschlossenen silbe ausfallende Laut $l$. Diese Erscheinung bildet eigentlich einen Übergang zur qualitätsmässigen Lautveränderung; im Hinblick darauf, dass es sich um einen Konsonanten handelt, der damit keine silbenschliessende Rolle mehr spielt, ist es jedoch zweckmässig, das Verhalten dieses Lautes hier zu behandeln. Der Ausfall des $l$ in einer geschlossenen Silbe (im Wortinnern und am Wortende) war im Altungarischen eine ziemlich allgemeine Erscheinung, die auch in den heutigen ungarischen Mundarten häufig vorkommt: szalma 'Stroh' $>$ szouma $>$ szóma; volt 'war' $>$ vout $>$ vót; volna '(es) wäre' $>$ vouna $>$ róna usw.

Es ist sehr schwer, den eigentlichen Grund für den obigen Ausfall des $l$ anzugeben. Am ehesten benutzt die Sprache diesen Ausfall vielleicht zur Vermeidung von Konsonantenhäufungen am Wort- und am Silbenende (föld 'Erde, Feld' > fö̈id, volt > vount, zöld 'grün'>zöüd usw.). Vom phonetischen Gesichtspunkt aus bereitet es keine Schwierigkeit, die Veränderung $l>u, \ddot{u}$ zu erklären; zur Aufklärung der sprachlichen Funktion derselben sind jedoch weitere Untersuchungen erforderlich.

\section{IV}

1. Hinsichtlich des Zusammentreffens zweier Vokale n a c h d e r e r s t e n S il be stellen wir folgendes fest:

- wenn ein kurzer Vokal neben einen langen gelangt, dann fällt unabhängig von der Reihenfolge der Laute stets der kurze Vokal aus: z.B. kovács 'Schmied' > koács $>$ kács,

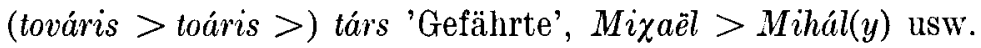
(Horger, M Ny XXV (1929) 255; Csüry, M Ny XXV (1929) $344)$;

- beim Zusammentreffen von zwei kurzen Vokalen fällt im allgemeinen der erste aus: z.B. gazda-asszony $>$ gazdasszony 'Wirtin, Hausfrau', tudni-illik 'das heisst' $>$ tudnillik; Ausnahmen gibt es auch hier: ${ }^{*} k i$-irit $>$ ki rit $>$ rit $\sim$ ritt 'ausrotten', të ëgyügyü > $>\ddot{e}$ gyüqgü 'einfältig' usw. 
Der Grund für die skizzierte sprachliche Veränderung liegt in der Gewährleistung der kontinuierlichen Lautbildung im Laufe des Gesprächs. Das Einschliessen der Silbengrenze zwischen zwei Vokale (der Hiatus) stört nämlich die Kontinuierlichkeit der Artikulation; deswegen strebt die Sprache nach dessen Elimination (s. E. Itkonen, Kieli ja sen tutkimus S. 171).

2. Für den Ausfall des kurzen Vokals der zweiten offenen Silbe finden wir zahlreiche Beispiele im Ungarischen, angefangen von den ältesten Zeiten, der Zeit der Urungarn (z.B. ${ }^{*}$ szerel $\ddot{s} m \ddot{8}>$ szerelmü $>$ altung. szerelm ${ }^{\ddot{u}}$ ) bis in unsere Zeit. Der Ausfall kann sich auf einen beliebigen kurzen Vokal beziehen (s. 1222: Zuluga > szolga 'Diener'; um 1200: Zecuseu (ว: szeküsëü) > szökcse > szöcske 'Grashüpfer'; (slaw. palica >) pálca 'Stab'; um 1200: Tocota (Ortsname) > Takta; 1121: Beseneu- (э: besenyëü) > Besnyö; um 1320: chudalatus $>$ um 1350: chudaltus ( 0 : tšudaltuš usw.) und zwar sowohl in der alten als auch in der heutigen Sprache.

Der Grund für den Ausfall der zweiten offenen Silbe ist in der Betonung zu suchen. Im Falle einer dynamischen Betonung nimmt die Intensität stark ab; dieser Tatsache fällt der kurze Vokal der offenen Silbe zum Opfer (Bárczi, Ht. ${ }^{2} 79$ ). Im Zusammenhang mit der Lautelision im Wortinnern haben auch viele andere Forscher ein ähnliches Prinzip vertreten (vgl. E. Itkonen a.a.O. 172-3), in erster Linie auf Grund einer Untersuchung finnischer, estnischer, wepsischer und lappischer Belege.

Sehr interessante Bemerkungen hinsichtlich des Zustandekommens der Tendenz von zwei offenen Silben stammen von J. Balázs (NytudÉrt 58 (1967) 70). Er wirft die Frage auf, ob die Ursache für den Lautausfall wirklich und ausschliesslich in der dynamischen Betonung liegt, oder ob man nicht auch an eine andere Erklärung denken könnte. Gekonnt weist er darauf hin, dass wir die Lautelision im Wortinnern von den dreisilbigen Wörtern finnisch-ugrischen Ursprungs, die mit Endungen uralter Herkunft (z.B. $\left.{ }^{*} \dot{a} l s-m s,{ }^{*} f e ̈ s z \ddot{8}-k \ddot{8}\right)$ versehen sind, ableiten können, und dass "d e $r$ A u sf a 11 des Vokals der zweiten offenen Silbe in den obigen Formen durcheinen Schw und 
des stam ma uslauteszuerklären is t» (a.a.o. 72).

Jenes Moment der Untersuchungen von J. Balázs, in dem er einen Zusammenhang zwischen der Tendenz der zwei offenen Silben und dem Schwund der Stammauslautvokale sieht, ist ausserordentlich lehrreich. Ich glaube jedoch, dass der Gang der Entwicklung eben der umgekehrte gewesen sein dürfte, das heisst die Lautelision im Wortinnern ist dem Ausfall der Stammauslautvokale vorangegangen. So müssen beide Erscheinungen auf einen oder mehrere gleiche Gründe zurückgehen. Dieser gemeinsame Grund ist ganz gewiss in den Gesetzmässigkeiten der dynamischen Betonung zu suchen. Auf die wichtige Rolle der Betonung der ersten Silbe in einer Sprache ist von mehreren Autoren im Zusammenhang mit ihren sprachlichen Forschungen im Finnischen, Estnischen, Lappischen und Wepsischen hingewiesen worden (E. Itkonen a.a.O. 157, 172-3; E. A. Tunkelo, Vepsän kielen äännehistoria. H:ki, 1946. SKSToim. 228. 718; M. Rapola, Suomen kielen äännehistorian luennot. H:ki, 1966. SKSToim. 238. 486 usw.).

3. Hinsichtlich der Stellung zwischen zwei Vokalen sehen wir sowohl in der altungarischen Zeit als auch in der heutigen Sprache, in erster Linie aber in den Mundarten, den Ausfall des $\beta$-, beziehungsweise des aus ihm entwickelten $v$-, des $-j-(i-)$ und des $-\gamma-,-h-$. Den Schwund weisen wir durch die folgenden Beispiele nach:

$-\beta-,-v>\varnothing:$ 1055: cues (c: küës), tue (c: tüē), um 1200: tilutoa (э: tilutoā) usw.; heutige dial. Formen: sü$v e g>$ süög $\sim$ süeg, üveg $>\ddot{u} e g \sim \ddot{o e g}$ usw.

$-j-(-i-)>\emptyset:$ altürk. qüjïn > 1138/1329: Kinusti, 1300: kynaal (vgl. kin 'Pein'); (bajos 'schwierig' $>$ ) dial. baos, (olyan 'solcher' $\sim$ ojan $>$ ) oan usw.

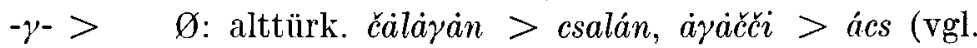
1214: Soluhan; 1233: Alch) usw.

-h- > Ø: Abraham > um 1200: abraam, 1171: Abram, heute Ábrány (Ortsname) usw.

Der Ausfall der behandelten Laute in der Lautfolge des Wortes kommt durch einen Hiatus zustande. Diese Erschei- 
nung müssen wir als reziproke Lautentwicklung, als Gegenpol zu jener Lauteinfügung $(-\beta-,-r-,-j-(-i-),-h-)$ bewerten, die der Beseitigung des Hiatus dient.

4. Die Konsonantenhäufungen im Wortinnern werden in der Regel auf folgende Weise aufgelöst:

- in den Lautverbindungen $-s v-,-s z v-,-l v-,-d v-,-t v-,-r v-$ schwindet in der Regel das $v$. Dies können wir hauptsächlich durch unsere alten sprachlichen Angaben belegen: 1226: Asuantheu (э: āsvāńtëü) 1263: Asantu (ว: āsāńtũ); 1222: Baluanus (o: bālvāńuš) 1211: Balanus ( : bālāńuš) usw. Ähnliche Ausfälle kamen auch bereits in urungarischer Zeit vor (vgl. z.B. finn. talvi $\sim$ ung. tele-, téle-; finn. survoa $\sim$ ung. szúr usw.);

- ebenfalls mit einem Ausfall ist bei einer dreifachen Konsonantenhäufung zu rechnen, wenn das $-v$ - als dritte Komponente auftritt: 1234/1364: Erezthwen > 1268: Erezteyn; estvel > estve > este, körtvél $(y)>$ körtély $>$ körte;

- von den Komponenten dreifacher Konsonantenhäufungen, die kein $v$ enthalten, fällt meist die mittlere aus (z.B. $-l m b->-l b-,-r l v->-r v-,-m l h->-m h-,-n t n->-n n-,-m b j->$ $-m j-,-n t g->-n g-,-n l d->-n d-,-r j f->-r f-$ usw. Selten kommt es dagegen vor, dass der erste Konsonant ausfällt (z.B. -lmb$>-m b-,-l m n->-m n-\mathrm{usw}$.).

5. Wir haben, wenn auch nicht sehr viele, Belege dafür, dass die nach einem Konsonanten stehende Lautverbindung $t+i \mathrm{zu} t y(=t)$ wird: z.B. bestia > bestye 'ludericht', ostia $>$ ostya 'Oblate' usw. Hier erfolgt eigentlich eine Verschmelzung; als Endergebnis der Entwicklung geht jedoch auf dem Wege der Palatalisierung des $t$ das Vokalelement $i$ verloren.

\section{V}

1. Hinsichtlich der i m a b s o l u t e n W or t a u s la u t erfolgenden Lautveränderungen gehen wir zunächst auf den Schwund der Vokale ein.

a) Die älteste der hierher gehörenden Erscheinungen ist vielleicht das Schwinden der Stammauslautvokale. Es ist bekannt, dass die kurzen Vokale oberer Zungenstellung 
$(i, ? i, u, \ddot{u})$ im Stamm- oder Wortauslaut unserer zwei- und mehrsilbigen Wörter aus der urungarischen Zeit bis zum Beginn des 13. Jahrhunderts im Ungarischen schwinden und nur vor gewissen Endungen (z.B. ház: háza-t, háza-k, háza-m, háza-s usw.) bestehen bleiben.

Das Schwinden der Stammauslautvokale wird von Bárczi durch Reduktion und von I. Papp durch Lautelision unter Mitwirkung gewisser funktioneller sprachlicher Gründe erklärt (vgl. I. Papp, UKH 127-9).

b) In einer verhältnismässig grossen Zahl von Belegen finden wir einen Ausfall der Laute $-a,-e$ am Wortende: apacsin (vgl. ukrain. opačina), beszéd (vgl. slaw. *bešeda), bolt 'Gewölbe' (? ital. volta), kolesz (slaw. kolesa), kolbász (slaw. *kalbasa), kamat (slaw. kámata), ladik (slaw. *ládijka), lapát (slaw. lopata), palánt (lat. planta) usw. (vgl. TESz.; SzófSz.).

Der Schwund der Endlaute $-a,-e$ in diesen Angaben kann eigentlich durch eine falsche Artikulation erklärt werden. Ein Teil der sprechenden Personen hat die Laute $-a,-e$ am Wortende mit den besitzanzeigenden Personalsuffixen $(-a,-e)$ identifiziert und eine falsche Nominativform abstrahiert.

c) In unseren Ortsnamen mit der Nachsilbe -földe, -telke, -háza, -halma, -laka, -mente, -köze, -szöge, -falva fällt das besitzanzeigende Personalsuffix $-a,-e$ vor dem adjektivierenden $-i$ aus: z.B. Gutorfölde $>$ gutorföldi, Jánostelke $>$ jánostelki, Pálháza > pálházi usw.

d) Der Ausfall des $a$ am Wortende einiger Wörter (z.B. lat. balaena > ung. 1538: balÿn, ? dt. halleparte > ung. alabárd) ist durch die Analogiewirkung der Lautformen des Fischnamens balin, bzw. durch bárd erklärbar.

2. Die Fälle von Konsonantenschwund sind die folgenden:

a) Gelangen die Laute $-v(-\beta),-\gamma(-h)$ und $-j(-i)$ in absoluten Wortauslaut, so schwinden sie im Laufe der urungarischen Zeit durch Vokalisierung, d.h. sie werden in der Regel zu $-u,-\ddot{u}$, wobei sie mit dem Stammauslautvokal einen Diphthong bilden. Z.B. urung. ${ }^{*}$ kivi $\sim{ }^{*}$ ki $\beta>$ altung. kiü $>>$ $k o$, slaw. postavz $>$ ung. poszta $\beta>$ postau $>$ posztó usw. Auf ähnliche Weise wird - ohne Ausnahme - das urungarische?' 


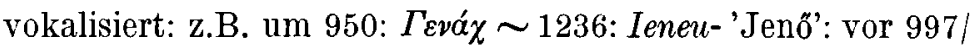
1109: $\sigma \alpha \mu \tau \alpha \dot{\gamma}($ ( : sảmta் $\gamma) \sim$ 1109: zamtou (o: sảmtou) 'Szántó' usw. Der Laut $-j(-i)$ hat schon in der urungarischen Zeit mit dem vor ihm stehenden Stammauslautvokal einen Diphthong gebildet, in vielen Fällen ist sogar eine Monophthongierung vor sich gegangen (s. Bárczi — Benkő - Berrár, M NyT. 169). Diese Erscheinung zieht sich auch bis in die altungarische Zeit hin (z.B. 1216: Keyc (э: këik) > um 1240: Kec (э: kēk) 1337: kyk- (॰: kīk) 'blau'; slaw. gnojz > ung. ganaj gané 'Dünger'; vgl. nach 1416/um 1450: ganéual) vereinzelte Fälle davon sind sogar in den heutigen Mundarten anzutreffen (vgl. taraj $\sim$ taré, paraj $\sim$ paré).

In $\mathrm{Silb}$ e n e d st e $11 \mathrm{ung}$ stellen wir dieselbe Entwicklung fest.

b) Vereinzelt kann der Auslautkonsonant der ursprünglichen Nominativform verschwinden, wenn er hinsichtlich seiner Lautform mit irgendeiner häufigeren Fügung übereinstimmt. Hierzu wollen wir uns einige Beispiele ansehen.

$-t>\varnothing$ : damaszt > damasz; furmint > furmin; (dt. Ringlotte $>$ ) ringló; (rumän. roṣcát $>$ ) ung. roska, róska; (slaw. podusto, podúst >) ung. paduc; (slaw. pöstāt, postatz, ung. pászta; (bayer.-österr. adutt $>$ ) ung. adu usw.

$-d>\varnothing:($ slaw. jagnjéd >) jegenye; (slaw. medvédb >) medve; (slaw. rásad >) rásza 'Sämling, Steckling' usw.

$-k>\varnothing$ : (türk. *biličäk >) bilincs; (slaw. čárdāk >) csárda; (slaw. polturák $>$ ) poltura 'Schilling' usw.

$-(u) s,-(o) m>\varnothing:$ Adrianus $>$ Adorján, Martinus $>$ Márton, (lat. cymbalum $>$ ) ung. cimbalom $\sim$ 1855: cymbál, 1865: cymbal usw.

Der Grund für alle diese Lautausfälle liegt darin, dass infolge eines falschen Sprachgefühls die sprechenden Personen die am Wortende stehenden Laute für Endungen hielten: so wurde das Element - $t$ für ein Objektsuffix gehalten, das - $d$ für ein besitzanzeigendes Personalsuffix der zweiten Person Singularis, das $k$ für ein Pluralzeichen; das $-(u) s,-(o) m$ dagegen wurde als diminutivbildend und als besitzanzeigendes Personalsuffix (Sg. 1. P.) empfunden und vom Wortende abgetrennt. 
c) Der Ausfall des $-r,-n,-n y$ am Wortende ist eine ausserordentlich seltene Erscheinung: z.B. akkor $\sim$ akko, mikor $\sim$ mikó, hamar hama, hány há, há usw. Im Wortinnern und im Wortauslaut ist dies häufiger anzutreffen: z. B. um 1200: zenuholmu (॰: senüholmu) 1417: Zyhalom, heute Szihalom, 1300: sumha 1516-19: soha; pénz > dial. péz, piz; ferslóg > fëslag, fëslóg; gardrób > 1835: gadrob, dial. kadrop usw.

Über den Ausfall des $r$ s. oben III/3.

d) Nach einem Konsonanten fällt das - $t$ und das $-d$ in vielen oft gebrauchten Wörtern aus: z.B. miért $\sim$ miịér, azért $\sim$ azér $;$ most $\sim$ mos, majd $\sim$ maj, megint $\sim$ megin usw.

Die Ursache für den Schwund dürfte in dem Streben nach Energieeinsparung liegen und könnte auch zur Vermeidung einer absoluten Wortauslautstellung oder zur Vermeidung der an der Wortgrenze auftretenden Konsonantenhäufung erfolgt sein.

e) Ebenfalls vereinzelt tritt die Auflösung gewisser Konsonantenhäufungen am Wortende dadurch auf, dass einer der sich häufenden Konsonanten ausfällt: palack $\sim$ dial. palac, tarack $\sim$ tarac, barack $\sim$ barac.

Das Ziel der Lautelision ist hier die Eliminierung von Erscheinungen, die mit den strukturellen Eigenheiten der ungarischen Sprache nicht vereinbar sind; ihre Ursache ist in der Wirtschaftlichkeit zu suchen.

\section{VI}

Die Behandlung der Arten von Lautschwund wäre vollständig, wenn wir die durch ausserordentlich häufige Wortverwendung entstehenden Elisionen ausser acht liessen, welche besonders in den Höflichkeitsformeln auftreten (isten áldja > sten álgya 'leben Sie wohl'), ferner in den durch Emphase verursachten Lautelisionen (akasszanak fel! 'soll man mich aufhängen!' > 'kasszanak fel!; egye ki a fene! > 'gye $k$ ' a fene! 'der Teufel soll es holen!'), in den Fällen von Lautveränderungen durch spielerische Kosenamen (Évica > Vica, Ilonci > Lonci) und Haplologie (közvetetlen > közvetlen 'unmittelbar; freundlich'). 
Abschliessend kann gesagt werden, dass die obige Zusammenstellung alle diejenigen Fälle von Elisionen enthält, die die Lautform des im Satz auftretenden Wortes gestalten können; nur in sehr seltenen individuellen Fällen treten Ausnahmen auf. Eine synchrone Untersuchung der Veränderungen zeigt, dass mit Ausnahme einiger Fälle (das Schwinden von Stammauslautvokalen und von schon ausgestorbenen Konsonanten, z.B. $\gamma, \beta$ ) diese auch in der heutigen Sprache, in erster Linie in den Mundarten anzutreffen sind. In der Alltagssprache und in der Literatursprache ist der Schwund der Stammauslautvokale, die Auflösung der Konsonantenhäufungen und der altungarische Ausfall von Vokalen der zweiten offenen Silbe und gewissen Konsonanten $(\gamma, v, j, h)$ zu einer allgemeinen Erscheinung geworden; die übrigen Veränderungen treten nur vereinzelt in der Literatur, in der Alltagssprache oder in der Volkssprache auf.

Aus den bearbeiteten Belegen - die hier nicht veröffentlicht werden können - geht hervor, dass die Fälle von Schwund in erster Linie auf eine unbetonte oder auf eine absolute Wortauslautstellung konzentriert sind. Dies beweist auch, dass die erste betonte Silbe im Ungarischen um jeden Preis die Wahrung der eigenen Position anstrebt; die unbetonte Stellung bietet dagegen mehr Möglichkeiten für einen Lautschwund. Das Streben nach Ökonomie spielt eine bedeutende Rolle; dies zeigt sich hauptsächlich in der Vermeidung des Hiatus (gazda-asszony > gazdasszony, helsin$k i+i>h e l s i n k i)$, ferner aber auch bei der sogen. reziproken Lautentwicklung (Analogie), die sich im Hintergrund mehrerer Lautveränderungen abspielt (z.B. der Ausfall gegenüber hiatusfüllenden Lauten und das Zustandekommen von Konsonantenhäufungen im Wortanlaut). Eine mehr oder weniger wichtige Rolle spielt - wie wir sahen - die Wortgrenzverwechslung, die analoge Wirkung der Lautform je eines Wortes, die Emphase, sowie die grosse Frequenz gewisser Wortformen und die Palatalisierung.

Natürlich spielen die physiologisch-phonetischen Lautent- 
wicklungen (Schwund des $l>\emptyset,-\beta,-h,-j$ am Wortende) eine bedeutende Rolle, z.B. im Umformen von offenen und geschlossenen Silben nach Bedarf (szalma > > szóma; palica > púlca; számtày $>>$ szántó), obgleich manchmal die Zusammenhänge dieser Veränderungen in bezug auf die Systemlehre im Unklaren bleiben.

Das Ziel aller dieser Veränderungen ist eigentlich die elastische Wahrung der ursprünglichen Lautstruktur und des Aufbaus des im Satz fungierenden Wortes. Wenn auch scheinbar Abweichungen von diesem Prinzip vorkommen, wie z.B. der Schwund der Stammauslantvokale, wodurch die Lautform unserer zweisilbigen Wörter finnisch-ugrischen Ursprungs wesentlich verändert worden ist, so wirkt dennoch auch hier im Hintergrund die folgende Tendenz, dic bald imstande sein wird, die "Irrtümer" der Sprachentwicklung zu korrigieren. Hierdurch ist es gewiss zu erklären, dass die Sprache den Ausfall der Stammauslautvokale in der urund altungarischen Zeit durch eine Verkürzung der langen Stammauslautvokale ersetzte; diese wiederum wurden durch lange Vokale ersetzt, die sich aus den Diphthongen entwickelt hatten. Die Diphthonge dagegen wurden durch Konsonantenelemente substituiert, die zur "Vokalisierung" bereit sind $(-\beta,-\gamma,-j)$. So können die Laute vom Gesichtspunkt der Lautveränderungen eigentlich in drei, sogar vier Gruppen eingeteilt werden: Vokale, konsonantenartige Vokale (z.B. $-u,-\ddot{u})$, Konsonanten und vokalartige Konsonanten.

Beim Durchbruch und bei der Verbreitung der Tendenz der zwei offenen Silben dürfte ganz gewiss die in der urungarischen Zeit (s. Rédei, NyK LXVI 256-259; Hajdú, BUNỵ 110) erfolgte Entwicklung eine Rolle gespielt haben, die aus der Vereinfachung einer Gruppe (vgl. ${ }^{*}-m p->-b-{ }^{*}{ }_{-m}-m$, ${ }^{*} n t->-d-,{ }^{*}-\eta k,-\eta g^{-}>-g-,-n c^{-}>-g y-$, ? *-ks'- > sz-; finn. kumpua- $\sim$ ung. habo-; finn. anta- $\sim$ ung. ado-, adu-, finn. kunta $\sim$ ung. hada-; finn. onte- $\sim$ ung. odu; finn. tunke- $\sim$ ung. dugo-; wog. kōins $\sim$ ung. húgy 'Stern'; wog. D tüks $\sim$ ung. $\delta s z)$ von Konsonantenverbindungen im Wortinnern bestand; diese Entwicklung hat in der Strukturierung der Wörter eine Veränderung hervorgerufen (geschlossene + offene Silbe > 
offene + offene Silbe, d.h. CVC-CV > CV-CV, bzw. VCCV > V-CV), welche die Zahl der aus der ugrischen Zeit ererbten und wahrscheinlich schon in reichem Masse vorhandenen Stämme rom Tỵp CV-CV noch weiter vergrösserte. Zur relativen Wiederherstellung und teilweisen Erhaltung des gestörten Gleichgewichts waren die als Ergebnis der Tendenz der zwei offenen Silben entstehenden neueren Wortformen vom Typ CVC-CV und VC-CV geeignet.

Nach dem Überblick über die obigen Lautveränderungen sehen wir, dass diese bei der Gestaltung der Wörter eine bedeutende Rolle spielen und in erster Linie deren Verkürzung und quantitative Abnahme regeln.

Istvíx NyiRkos 